\title{
Violencia en el Ámbito Universitario: El caso de la Universidad Nacional de Colombia
}

\author{
Diana Amórtegui-Osorio \\ Fonoaudióloga. M.Sc. Salud Pública. Tel 2256848. E- mail: prindao@hotmail.com
}

Recibido 15 Agosto 2003/Enviado para Modificación 10 Febrero 2004/Aceptado 5 Junio 2005

\section{RESUMEN}

Objetivo Estimar la prevalencia de creencias, percepciones, actitudes y prácticas en su rol de testigo y posible actor de disturbios, en la comunidad estudiantil de la Universidad Nacional de Colombia, sede Bogotá.

Métodos Se aplicó una encuesta a 500 estudiantes de pregrado en lugares dentro del campus (cafeterías, salas de estudio, lugares de encuentro, bibliotecas). La encuesta tenía 18 variables agrupadas según el tipo y grado de violencia. La información obtenida se digitó en una base de datos Access 2000 y se procesó mediante el paquete SPSS 8.0 para Windows.

Resultados De un total de 496 encuestas útiles, 271 (54,5\%) resultaron no agresores, $163(32,8 \%)$ agresores tipo 1 y 63 (12,7\%) agresores tipo 2. Se encontraron relaciones significativas entre los tipos de violencia y el sexo masculino, estado civil, promedio académico y el número de parejas íntimas, mas no con la edad ni la pertenencia a una Facultad.

Conclusiones Los medios violentos de expresión fueron pocos o nada aceptados por la mayoría de los encuestados quienes prefieren expresar sus opiniones y necesidades a través de los representantes estudiantiles y grupos de trabajo. Sin embargo, las personas que participan en los disturbios afirmaron que no existen medios suficientes o alternativos de expresión, justificando los hechos violentos en ciertas situaciones. Los agresores tienden a ser de género masculino y creen que las acciones violentas son más aceptables.

Palabras Claves: Violencia, universidad, estudiantes, Colombia (fuente: DeCS, BIREME).

ABSTRACT

Violence in the university environment: The case of the National University of Colombia 
Objective To estimate the prevalence of beliefs, attitudes, perceptions and practices in the roles of witness and possible actor in riots, among the student community of the National University of Colombia in Bogotá.

Methods A survey was applied to 500 undergraduate students in places within the university campus (cafeterias, study rooms, meeting places libraries). The survey comprised 18 variables which were grouped according to type and degree of violence. The information obtained was included in an access 200 database and analyzed with the SPSS 8.0 software for Windows.

Results From 496 useful surveys, 271 students (54.5\%) were identified as non-aggressors, 163 (32.8) as type 1 aggressor and 63 (12.7\%) as type 2 aggressors. Significant relationships were found between the types of violence and the male sex, marital status, academic average, number of intimate couples but not with age nor with studying a given career.

Conclusions Violent means of expression had little or no acceptance at all by the majority of students surveyed, who prefer their opinions to be expressed through the student representatives or in working groups. However, persons participating in riots state that the available means or alternatives of expression are not enough, justifying their violent acts in certain situations. The aggressors tend to be males and believe that violent actions are more acceptable.

Key Words: Violence, universities, students, Colombia (source:MeSH, NLM)

$\mathrm{L}$ a violencia, definida como la amenaza o uso de la fuerza física o poder en contra de otras personas, contra uno mismo, contra objetos o contra un grupo o comunidad (1) es un problema de primer orden en Colombia. Además de las lesiones físicas, la violencia genera enlas víctimas y testigos efectos psicológicos relacionados con el estrés postraumático, ansiedad crónica, depresión, pérdida de la autoestima, trastornos del sueño, problemas de apetito, múltiples enfermedades psicosomáticas, alcoholismo y en algunos casos suicidio (2).

Las conductas y actos violentos se presentan en gran número de instituciones, incluidas la Universidades, produciendo daños materiales, ausentismo, y personas lesionadas o muertas. Sin embargo, en el contexto nacional son escasos los estudios que aporten evidencia empírica al conocimiento sobre violencia estudiantil. Gran parte de las investigaciones se enfocan en la violencia originada por el conflicto armado y la delincuencia común.

La violencia no es un fenómeno aleatorio. Múltiples estudios muestran que tiende a concentrarse en ciertos lugares, momentos y entre cierta población (3). Los factores culturales son comúnmente señalados como factores 
determinantes de cualquier tipo de violencia. Se entiende como cultura el conjunto de normas, actitudes, valores y creencias transmitidos y compartidos por un grupo social que le da coherencia a la forma como sus miembros actúan, interpretan y responden a las circunstancias (4). Las personas involucradas en hechos violentos tienden a creer que la violencia está bien y que se justifica en ciertas situaciones (5-6).

En el presente estudio se intentó una aproximación a las creencias, actitudes y prácticas que pudieran tener los estudiantes frente a los actos violentos en su medio.

\section{MATERIALES Y MÉTODOS}

Se elaboró y aplicó una encuesta a 500 estudiantes matriculados en carreras de pregrado de la Universidad Nacional de Colombia-UNC, Sede Bogotá en octubre y noviembre de 2001.

Cuadro 1. Clasificación de la violencia estudiantil en la UNC,

\begin{tabular}{|c|c|c|}
\hline \multicolumn{2}{|c|}{ Violencia Estudiantil } & \multirow{2}{*}{ Expresiones Violentas } \\
\hline Grado & Tipo & \\
\hline No Agresor & $\begin{array}{l}\text { No manifiesta } \\
\text { ningún tipo de } \\
\text { comportamiento } \\
\text { violento }\end{array}$ & Ninguna \\
\hline Agresor Tipo 1 & $\begin{array}{l}\text { Chifla /Burla de } \\
\text { otro Amenaza } \\
\text { /Intimida }\end{array}$ & $\begin{array}{l}\text { Chiflar a un compañero, burlándose de él } \\
\text { Chiflar a la Policía } \\
\text { Amenazar verbalmente, insultar a un } \\
\text { estudiante de la U.N. } \\
\text { Amenazar verbalmente, insultar a un } \\
\text { profesor de la U.N. } \\
\text { Enviar cartas amenazando a alguien }\end{array}$ \\
\hline Agresor Tipo 2 & $\begin{array}{l}\text { Daño Propiedad / } \\
\text { Bienes } \\
\text { Pone en peligro } \\
\text { su integridad y la } \\
\text { de otros }\end{array}$ & $\begin{array}{l}\text { Escribir mensajes en las paredes } \\
\text { Destruir pupitres } \\
\text { Quemar pupitres } \\
\text { Destruir ventanas } \\
\text { Destruir Laboratorios } \\
\text { Destruir Casilleros } \\
\text { Golpear, pegar o hacer daño físico a un } \\
\text { estudiante de la universidad } \\
\text { Golpear, pegar o hacer daño físico a un } \\
\text { profesor de la universidad } \\
\text { Lanzar piedras a la policía } \\
\text { Lanzar petardos a la policía } \\
\text { Encapucharse para enfrentar a la policía } \\
\text { Quemar buses y/o carros } \\
\text { Utilizar armas de fuego contra otros dentro } \\
\text { de la Universidad }\end{array}$ \\
\hline
\end{tabular}


La Universidad Nacional es una institución pública. La muestra de estudiantes fue escogida por conveniencia. Se recolectó información relacionada con la edad, género y Facultad a la cual pertenecían los encuestados.

Para la exploración de creencias, actitudes y prácticas relacionadas con hechos violentos dentro del campus se diseñó una clasificación del tipo y grado de violencia en la cual se agruparon las 18 variables empleadas en la encuesta. Se obtuvieron tres tipos o grados de violencia que permiten diferenciar a los no agresores de los agresores, como se indica en el Cuadro 1.

El instrumento éste fue ajustado por medio de una prueba con 20 personas (14 estudiantes de pregrado, 4 de maestría y 2 ex-alumnos de la U.N.). La información obtenida se digitó en una base de datos Access 2000 y se procesó mediante el paquete SPSS 8.0 para Windows.

\section{RESULTADOS}

De un total de 496 encuestas útiles, 271 (54,5\%) resultaron no agresores, 163 (32,8 \%) agresores tipo 1 y 63 (12,7 \%) agresores tipo 2.

Violencia según género

Se encontró que los hombres han sido actores de todos los tipos de violencia con mayor frecuencia que las mujeres: 63,2 \% del tipo 1 y 82,5 \% del tipo 2 . Así mismo, la proporción de hombres se incrementa con la gravedad de la violencia. Se encontró una relación significativa entre el comportamiento violento y el género masculino (Tabla 1).

Tabla1. Distribución del grado de violencia por auto reporte, según género.

\begin{tabular}{|c|c|c|c|c|c|c|c|c|}
\hline \multirow{3}{*}{ GENERO } & \multicolumn{6}{|c|}{ TIPO DE VIOLENCIA } & \multirow{2}{*}{\multicolumn{2}{|c|}{ TOTAL }} \\
\hline & \multicolumn{2}{|c|}{ Tipo 0} & \multicolumn{2}{|c|}{ Tipo 1} & \multicolumn{2}{|c|}{ Tipo 2} & & \\
\hline & Frec. & $\%$ & Frec. & $\%$ & Frec. & $\%$ & Frec. & $\%$ \\
\hline Masculino & 94 & 34,7 & 103 & 63,2 & 52 & 82,5 & 249 & 50,3 \\
\hline $\begin{array}{l}\text { Femenino } \\
\text { No }\end{array}$ & 176 & 64,9 & 60 & 36,8 & 11 & 17,5 & 247 & 49,9 \\
\hline Respuesta & 1 & 0,4 & 0 & 0,0 & 0 & 0,0 & 1 & 0,2 \\
\hline TOTAL & 271 & 100,0 & 163 & 100,0 & 63 & 100,0 & 497 & 100,0 \\
\hline
\end{tabular}


Distribución de la violencia por grupos de edad

La distribución de los tipos de violencia siguió el mismo patrón que los grupos de edad totales. Los tipos de violencia 1 y 2 resultaron más frecuentes en las personas que tenían 18 a 21 años, con 57,4 \% y 58,7 \% respectivamente. Sin embargo, no se encontró asociación entre la edad y los comportamientos violentos (Tabla 2).

Tabla 2. Distribución del grado de violencia por auto reporte, según edad en años

\begin{tabular}{|c|c|c|c|c|c|}
\hline \multirow{3}{*}{$\begin{array}{l}\text { Edad } \\
\text { (Años) }\end{array}$} & \multicolumn{4}{|c|}{ Tipo de Violencia } & \multirow{2}{*}{ TOTAL } \\
\hline & \multicolumn{2}{|c|}{ Tipo 0} & Tipo 1 & Tipo 2 & \\
\hline & Frec. & $\%$ & Frec. \% & Frec. \% & Frec. $\%$ \\
\hline 16 y 17 & 21 & 7,8 & 4,3 & 6,3 & 32 \\
\hline 18 a 21 & 161 & 59,6 & 9357,4 & 3758,7 & 29158,8 \\
\hline 22 a 26 & 88 & 32,6 & 6238,3 & 2234,9 & 17234,7 \\
\hline TOTAL & 270 & 100,0 & 162100,0 & 63100,0 & 495100,0 \\
\hline
\end{tabular}

Violencia según estado civil

El 95,7 \% de los violentos tipo 1 y el 87,3 \% de los violentos tipo 2 eran solteros. En el grupo de no agresores, 94,8 eran solteros. Por lo contrario, sólo el 13,1 \% de los casados o que vivían en unión libre, resultaron ser agresores. Aunque estas frecuencias podrían estar relacionadas con las características de la población de estudiantes de pregrado, quienes en su mayoría son solteros, la prueba de Fisher identificó una relación significativa entre el estado civil y el grado de violencia $(\mathrm{p}=0.025)$.

\section{Violencia según Facultad}

Se encontró que en todas las Facultades predominan los no agresores (violencia tipo 0). La Facultad que presenta mayor número de no agresores fue Ciencias Humanas con un 18,5 \% del total. Aquellos actores más violentos o que manifestaron tener comportamientos más violentos (tipo 2) se ubicaban en las Facultades de Ingeniería, Derecho y Ciencias Humanas, cada una con $15,8 \%$ del total de actores. Los agresores tipo 1 se encontraron en mayor proporción en la Facultad de Ingeniería, con 24,5 \% del total. Sin embrgo, esta Facultad también tiene el mayor número de estudiantes de pregrado matriculados: 22,8 \% del total de matriculados para el periodo 2000-I (7). A pesar de lo anterior, no se encontró relación significativa entre la pertenencia 
a una Facultad y las proporciones de agresores (Pearson chi $^{2}=31.1925$ $\mathrm{p}=0.053$ ).

Violencia según promedio académico

El 52,9 \% de los no agresores (violencia tipo 0) tuvo un promedio académico entre 3,6 y 4,0 sobre 5,0. El promedio académico con mayor frecuencia para el tipo de violencia 1 se ubicó entre 3,0 y 3,5, con 44,1\%. Para el tipo de violencia 2 el promedio académico se ubicó entre 3,0 y 3,5 con $49 \%$. Se encontró una relación significativa entre el promedio académico y los agresores $\left(\right.$ Pearson chi $^{2}=19.2743 \mathrm{p}=0.037$ ).

Violencia según número de parejas íntimas

El 82,6 \% de los no agresores mencionó haber tenido entre 1 y 5 parejas íntimas durante los cinco años anteriores. Igual se encontró para los tipos de violencia 1 y 2 . Se observó que los agresores suelen tener mayor número de parejas íntimas en comparación con los no agresores (Pearson $\mathrm{chi}^{2}=$ 29.7154; $\mathrm{p}=0.000$ ).

Aceptabilidad de formas de expresión en el campus

Los grupos de trabajo son considerados por el 32,9 \% de los no agresores como un medio muy aceptable de los estudiantes para expresar sus opiniones y necesidades. En los tipos de violencia 1 y 2 sólo el 19,4 \% y 6,5 \% respectivamente, consideraron este medio de expresión como muy aceptable.

Los representantes estudiantiles fueron considerados por el 20,7 \% de los no agresores como un medio de expresión muy aceptable; as \{i tambi $\{$ en lo consideraron el 16,4 \% de los violentos tipo 1 y el 5,1\% de los violentos tipo 2.

Del total de no agresores, 26,8 \% consideraron las asambleas como un medio muy aceptable de expresión, mientras que consideraron lo mismo sólo el 16,6 \% de los actores violentos tipo 1 y el 4,7 \% de los violentos tipo 2 .

Las marchas fueron consideradas muy aceptables por el 19,7 \% de los no agresores, $14,3 \%$ de los agresores tipo 1 y por el 6,1 \% de los agresores tipo 2. 
La aceptación de escribir mensajes en las paredes como forma de expresión de los estudiantes, fue considerada nada aceptable por el 29,6 \% de los no agresores, el 3,9 \% de los actores tipo 2 y el 10,3 \% de los actores tipo 1 .

Gritar frente a las oficinas de las directivas fue considerado como nada aceptable por el 28,1 \% del total de no agresores, 12,3 \% de los agresores tipo 1 y por el $13,7 \%$ de los agresores tipo 2 . Esta forma de expresión es calificada como aceptable por el 9,2\% de los agresores tipo 1 y por el 3,9\% de los agresores tipo 2 .

Tanto los agresores como los no agresores consideraron nada aceptable amenazar con hacer daño a otros; sin embargo, el grupo de agresores tipo 2, consideró poco aceptable y aceptable esta forma de expresión dentro del campus sólo en un 3,5 y 0,8 \% respectivamente.

Romper vidrios es una forma de expresión calificada como nada aceptable por la mayoría de agresores y no agresores; sin embargo, el 0,6 \% de los agresores tipo 2 consideran que es una forma muy aceptable de lograr lo que se quiere y manifestar inconformidad.

Dañar pupitres es considerado nada aceptable por el 51,9 \% de los no agresores y por el 31,2 \% de los agresores tipo 1 . Del total de agresores tipo 1 y 2 tan sólo el 0,2 \% consideran que dañar pupitres es una forma muy aceptable de manifestar inconformidad.

De los no agresores el 40,6 \% consideran que enfrentar a la policía con piedras es una forma nada aceptable de expresar inconformidad y lo mismo ocurre con el 21,2 \% de los agresores tipo 1. De los agresores tipo 2, el 3,1 $\%$ y 3,5 \% consideran esta forma de expresión como muy aceptable y aceptable respectivamente.

Prender fuergo a buses o vehículos como medio para expresar inconformidad es calificado por el 51,6 \% de los no agresores como nada aceptable, Esta misma consideración la tienen $29,1 \%$ de los actores violentos tipo 1. Los agresores tipo 1 y 2 consideran este medio aceptable en un 0,6 \% y 10,4 $\%$ respectivamente.

\section{DISCUSIÓN}

Aunque se dispone de poca información sobre violencia estudiantil, algunos estudios indican que la mayoría de los participantes son hombres, adultos jóvenes sin antecedentes judiciales (8). Un informe de la Asociación Ameri- 
cana de Psicología, sugiere que la presencia e influencia del grupo genera un proceso de contagio y continuo escalamiento de la intensidad y gravedad de las acciones. En este tipo de violencia, los testigos tienen especial influencia y se sabe que entre más temprano intervienen más logran cambiar el curso de los eventos.

Los medios violentos de expresión fueron pocos o nada aceptados por la mayoría de los encuestados quienes prefieren expresar sus opiniones y necesidades a través de los representantes estudiantiles y grupos de trabajo. Sin embargo, las personas que participan en los disturbios afirman que no existen medios suficientes o alternativos de expresión, justificando los hechos violentos en ciertas situaciones (6).

El diálogo y las negociaciones no son concebidos como un medio efectivo para alcanzar lo que se quiere. Se cree que los hechos violentos tienen mayor repercusión en las autoridades académicas, a medida que son más intensos o graves.

La mayoría de las personas encuestadas tenía la idea de que en todos los disturbios participan personas externas a la Universidad. Incluso aquellas que mencionaron haberse encapuchado para enfrentar a la policía con piedras y petardos, reportaron que en cerca de la mitad de los disturbios participan en los enfrentamientos con la policía personas que no tienen ningún vínculo con la Universidad.

En términos generales podría decirse que los agresores tienden a ser de género masculino, lo que concuerda con otros estudios en los que se encontró que la mayoría de los participantes son hombres adultos (8). Estos suelen estar en unión libre o casados, con relaciones más estables pero a la vez con mayor número de parejas íntimas, no tienen un desempeño académico sobresaliente, creen que las formas no violentas son menos aceptables sin llegar a rechazarlas y que las acciones violentas son más aceptables

Agradecimientos. A Joanne Klevens por el tiempo dedicado en la planeación y revisión del proyecto de investigación y por sus valiosas recomendaciones. A los profesores Mauricio Restrepo, Carlos Agudelo y Juan Carlos Eslava.

\section{REFERENCIAS}

1. Organización Panamericana de la Salud. Condiciones de Salud en las Américas. Publicación Científica No. 524. Washington, D.C.; 1990. 
2. Rosenberg M, Mercy J.A. Assaultive Violence. In: Last JM, Wallace RB. eds. Maxcy-Rosenau-Last Public Health and Preventive Medicine. Norwalk CT: Appleton \& Lange; 1992.

3. Mercy JM, Carroll P. New Directions in Violence Prediction: The Public Health Arena. Violence and Victims 1988; 3(4): 285-301.

4. Susser MW, Watson W, Hopper K. Sociology in Medicine. New York: Oxford University Press; 1985.

5. Orpinas P. Who is violent? Factors Associated with Aggressive Behavior in Latin America and Spain. Pan American Journal of Public Health 1999; 78: 232244.

6. Duque LF, Klevens J. Creencias, Actitudes y Prácticas Asociadas a la Violencia en Bogotá. Coyuntura Social 2000; 22: 187-202.

7. Eron L, Gentry JH, Schlegal P. Reason to Hope: A psychosocial perspective on violence and youth. American Psychological Association: Washington DC.; 1996.

8. Universidad Nacional de Colombia. Estadísticas e Indicadores de la U.N. Revista Oficina de planeación 2000; 4. 